\title{
Diophantine inequalities for the non-Archimedean line $\mathbb{F}_{q}((1 / T))$
}

by

\author{
Chin-Nung Hsu (Taipei)
}

1. Introduction. In 1946, Davenport and Heilbronn [3] adapted the Hardy-Littlewood method to prove that if $\lambda_{i}(i=1, \ldots, K)$ are non-zero real numbers, not all of the same sign, and if $\lambda_{1} / \lambda_{2}$ is irrational, then the values of

$$
\lambda_{1} x_{1}^{k}+\ldots+\lambda_{K} x_{K}^{k}
$$

as $x_{i}$ 's run independently through all natural numbers, are everywhere dense on the real line provided that $K \geq 2^{k}+1$. In the case $k=1$, Baker [1] (see also [11] and [13]) showed that for any positive integer $n$ there exist infinitely many primes $p_{1}, p_{2}, p_{3}$ satisfying the inequality

$$
\left|\lambda_{1} p_{1}+\lambda_{2} p_{2}+\lambda_{3} p_{3}\right|<(\ln p)^{-n},
$$

where $p$ denotes the maximum of $p_{1}, p_{2}, p_{3}$. More recently, Harman [5] showed that if $\alpha$ is a real number, then there are infinitely many ordered triples of primes $p_{1}, p_{2}, p_{3}$ for which

$$
\left|\alpha+\lambda_{1} p_{1}+\lambda_{2} p_{2}+\lambda_{3} p_{3}\right|<\left(\max _{j} p_{j}\right)^{-1 / 5+\varepsilon} .
$$

In the case $k \geq 2$, Ramachandra [11] (see also [12]) showed that when $K \geq 2^{k}+1$ if $1 \leq k \leq 11$ and $K \geq 2\left[2 k^{2} \ln k+k^{2} \ln \ln k+2.5 k^{2}\right]-1$ if $k \geq 12$, the values of

$$
\lambda_{1} p_{1}^{k}+\ldots+\lambda_{K} p_{K}^{k}
$$

as the $p_{j}$ 's run independently through all primes, are everywhere dense on the real line. The key to the Hardy-Littlewood method on the real line is

2000 Mathematics Subject Classification: Primary 11D75, 11J25.

Key words and phrases: Hardy-Littlewood method, Diophantine inequalities, Hua's lemma. 
the integral

$$
\int_{-\infty}^{\infty} \exp (x y)\left(\frac{\sin \pi x}{\pi x}\right)^{2} d x=\max \{1-|y|, 0\}
$$

In this paper, we study the Hardy-Littlewood method for the completion $\mathbf{K}_{\infty}=\mathbb{F}_{q}((1 / T))$ of the rational function field $\mathbf{K}=\mathbb{F}_{q}(T)$ at the infinite place, where $\mathbb{F}_{q}$ denotes the finite field with $q$ elements. We have a natural discrete valuation $|\cdot|$ on $\mathbf{K}_{\infty}$ defined by

$$
|f|=q^{\operatorname{deg} f}
$$

where $\operatorname{deg} f$ denotes the degree of $f \in \mathbf{K}_{\infty}$ at $T$, and set $\operatorname{deg} 0=-\infty$. Since $\mathbf{K}_{\infty}$ is complete under the non-Archimedean valuation $|\cdot|$ and the Pontryagin (self) duality $\widehat{\mathbf{K}}_{\infty}=\mathbf{K}_{\infty}$ holds (cf. Section 2), we have the following basic analogy:

$$
\mathbb{F}_{q}[T] \sim \mathbb{Z}, \quad \mathbf{K} \sim \mathbb{Q}, \quad \mathbf{K}_{\infty} \sim \mathbb{R} .
$$

Let $p$ be the characteristic of $\mathbb{F}_{q}$, let $\lambda_{1}, \ldots, \lambda_{D}$ be non-zero elements in $\mathbf{K}_{\infty}$ satisfying $\lambda_{1} / \lambda_{2} \notin \mathbf{K}$ and

$$
\operatorname{sgn} \lambda_{1}+\ldots+\operatorname{sgn} \lambda_{D}=0
$$

where $\operatorname{sgn} f \in \mathbb{F}_{q}$ denotes the leading coefficient of $f \in \mathbf{K}_{\infty}$. We show that if $p>d \geq 1$ and

$$
D \geq \begin{cases}1+2^{d} & \text { if } 2 \leq d<11 \\ 2\left[2 d^{2} \ln d+d^{2} \ln \ln d+2 d^{2}-2 d\right]+1 & \text { if } d \geq 11\end{cases}
$$

then the values of the sum

$$
\lambda_{1} P_{1}^{d}+\ldots+\lambda_{D} P_{D}^{d}
$$

as the $P_{i}$ 's run independently through all monic irreducible polynomials in $\mathbb{F}_{q}[T]$, are everywhere dense on the "non-Archimedean" line $\mathbf{K}_{\infty}$. In fact, we obtain a more explicit inequality in Theorem 2.1. In the proof of Theorem 2.1, the integral (cf. Lemma 2.2)

$$
\int_{\mathbf{K}_{\infty}} E(a f) \chi_{n}(a) d a= \begin{cases}1 & \text { if } \operatorname{deg} f<n \\ 0 & \text { if } \operatorname{deg} f \geq n\end{cases}
$$

plays a role entirely analogous to the integral (1) on the real line.

We studied the case $d=1, D=3$ in [8]. In the present paper, we attack this problem in the case when $d \geq 2$. In this situation, we need more additive theory of monic irreducible polynomials in $\mathbb{F}_{q}[T]$ (see, e.g., Theorems 4.3, 4.4, and 2.4).

2. The main theorem and definition. Let $\mathbb{F}_{q}$ be the finite field with $q$ elements. Let $p$ be its characteristic and let $\mathbb{F}_{p}=\mathbb{Z} / p \mathbb{Z}$ be the subfield of 
$\mathbb{F}_{q}$ with $p$ elements. Let $\psi_{0}: \mathbb{F}_{p} \rightarrow \mathbb{C}^{\times}$be the canonical additive character defined by

$$
\psi_{0}([c])=\exp \left(\frac{2 \pi i \cdot c}{p}\right),
$$

where $[c]$ denotes the canonical image of $c$ in $\mathbb{F}_{p}$. Let $\psi: \mathbb{F}_{q} \rightarrow \mathbb{C}^{\times}$be the additive character defined by $\psi(x)=\psi_{0}(\operatorname{Tr}(x))$ for all $x \in \mathbb{F}_{q}$ where $\operatorname{Tr}$ is the trace map from $\mathbb{F}_{q}$ to $\mathbb{F}_{p}$. Let $\mathbf{A}=\mathbb{F}_{q}[T]\left(\right.$ resp. $\mathbf{K}=\mathbb{F}_{q}(T)$ ) be the polynomial ring (resp. rational function field) with coefficients in $\mathbb{F}_{q}$. Let $\mathbf{A}_{+}$denote the subset of $\mathbf{A}$ consisting of all monic polynomials. Let $\mathbf{K}_{\infty}=\mathbb{F}_{q}((1 / T))$ denote the completion of $\mathbf{K}$ at the infinite place; in other words, for every $a \in \mathbf{K}_{\infty}$, if $a \neq 0$, then $a$ can be expressed as

$$
a=\sum_{i=d}^{-\infty} c_{i} T^{i},
$$

where $c_{i} \in \mathbb{F}_{q}$ and $c_{d} \neq 0$. The sign, degree, and absolute value of $a$ are defined by $\operatorname{sgn} a=c_{d}, \operatorname{deg} a=d$, and $|a|=q^{d}$. The residue of $a$ at the infinite place is denoted by $\operatorname{Res}_{\infty} f=c_{-1}$. The exponential map $E: \mathbf{K}_{\infty} \rightarrow \mathbb{C}^{\times}$is defined by

$$
E(a)=\psi\left(\operatorname{Res}_{\infty} a\right) .
$$

The exponential map $E$ is a non-trivial additive character from $\mathbf{K}_{\infty}$ to $\mathbb{C}^{\times}$ and the Pontryagin (self) duality $\widehat{\mathbf{K}}_{\infty}=\mathbf{K}_{\infty}$ is deduced by the bilinear map

$$
\mathbf{K}_{\infty} \times \mathbf{K}_{\infty} \rightarrow \mathbb{C}^{\times}, \quad(a, f) \mapsto E(a \cdot f) .
$$

In this paper, the Haar integral for $\mathbf{K}_{\infty}$ is defined to satisfy

$$
\int_{\operatorname{deg} a \leq-1} 1 d a=1 .
$$

This implies that

$$
\int_{\mathbf{K}_{\infty}} f(a) d(b a)=|b| \int_{\mathbf{K}_{\infty}} f(a) d a
$$

for all $b \in \mathbf{K}_{\infty}$ and continuous functions $f$ (with compact support). With these properties, we have the following basic analogy:

$$
\mathbf{A} \sim \mathbb{Z}, \quad \mathbf{K} \sim \mathbb{Q}, \quad \mathbf{K}_{\infty} \sim \mathbb{R}, \quad E \sim \exp .
$$

The main theorem of this paper is

TheOREM 2.1. Suppose that $d, D, m$ are positive integers and $\lambda, \lambda_{1}, \ldots$ $\ldots, \lambda_{D}$ are non-zero elements in $\mathbf{K}_{\infty}$ satisfying $\lambda_{1} / \lambda_{2} \notin \mathbf{K}, 2 \leq d<p$,

$$
\operatorname{deg} \lambda_{1}=\ldots=\operatorname{deg} \lambda_{D}=0,
$$

and

$$
\operatorname{sgn} \lambda_{1}+\ldots+\operatorname{sgn} \lambda_{D}=0
$$


Then if

$$
D \geq \begin{cases}1+2^{d} & \text { if } 2 \leq d<11 \\ 2\left[2 d^{2} \ln d+d^{2} \ln \ln d+2 d^{2}-2 d\right]+1 & \text { if } d \geq 11\end{cases}
$$

then there exist infinitely many positive integers $N$ for which there are

$$
\gg \frac{q^{(D-d) N}}{N^{D+m}}
$$

D-tuples $\left(P_{1}, \ldots, P_{D}\right)$ of monic irreducible polynomials with $\operatorname{deg}\left(\lambda_{i} P_{i}\right)=N$ and

$$
\operatorname{deg}\left(\lambda+\lambda_{1} P_{1}^{d}+\ldots+\lambda_{D} P_{D}^{d}\right)<-m \ln N+1
$$

where the implied constant depends only on $\mathbf{A}, \lambda, \lambda_{i}, d, D$, and $m$, but not on $N$.

REMARK 1. The complete proof of Theorem 2.1 is given in Section 5. In fact, if we define the value of $I_{j}(a)$ in $(3)$ to be

$$
I_{j}(a)=I\left(a T^{\Lambda-\operatorname{deg} \lambda_{j}} \lambda_{j}\right)
$$

where

$$
\Lambda=\max _{1 \leq j \leq D}\left\{\operatorname{deg} \lambda_{j}\right\}
$$

then without the condition (2), the statement of Theorem 2.1 is also true.

2. The choice of $N$ depends on $\lambda_{1} / \lambda_{2} \in \mathbf{K}_{\infty} / \mathbf{K}$ and this condition is used only in Lemmas 4.2 and 4.6. Combining this theorem and [8], Theorem 1.2 , we obtain

CONSEQUENCE 1. Under the hypothesis of Theorem 2.1, suppose $p>d$ $\geq 1$ and

$$
D \geq \begin{cases}1+2^{d} & \text { if } d<11 \\ 2\left[2 d^{2} \ln d+d^{2} \ln \ln d+2 d^{2}-2 d\right]+1 & \text { if } d \geq 11\end{cases}
$$

Then the values of the sum

$$
\lambda_{1} P_{1}^{d}+\ldots+\lambda_{D} P_{D}^{d}
$$

as the $P_{i}$ 's run independently through all monic irreducible polynomials in $\mathbb{F}_{q}[T]$, are everywhere dense on the non-Archimedean line $\mathbb{F}_{q}((1 / T))$.

Let $\mathfrak{M}$ be the subring of $\mathbf{K}_{\infty}$ consisting of $a \in \mathbf{K}_{\infty}$ with $\operatorname{deg} a \leq-1$ and let $\chi_{0}$ be the characteristic function of $\mathfrak{M}$; in other words, $\chi_{0}: \mathbf{K}_{\infty} \rightarrow \mathbb{R}$ satisfies

$$
\chi_{0}(a)= \begin{cases}1 & \text { if } a \in \mathfrak{M} \\ 0 & \text { otherwise. }\end{cases}
$$

Given any integer $n$, the function $\chi_{n}: \mathbf{K}_{\infty} \rightarrow \mathbb{R}$ is defined by

$$
\chi_{n}(a)=q^{n} \chi_{0}\left(a T^{n}\right) \quad \text { for } a \in \mathbf{K}_{\infty} .
$$


LEMMA 2.2. We have

$$
\int_{\mathbf{K}_{\infty}} E(a f) \chi_{n}(a) d a= \begin{cases}1 & \text { if } \operatorname{deg} f<n, \\ 0 & \text { if } \operatorname{deg} f \geq n .\end{cases}
$$

Proof. See [6], Theorem 3.5.

Let $p>d \geq 2, N$ be fixed positive integers. We define functions

$$
\begin{aligned}
& S(a)=\sum_{\operatorname{deg} P=N}^{\prime} E\left(a P^{d}\right), \quad I(a)=\frac{1}{N} \int_{y \in T^{N}+T^{N} \mathfrak{M}} E\left(a y^{d}\right) d y, \\
& S_{j}(a)=S\left(a \lambda_{j}\right), \quad I_{j}(a)=I\left(a \lambda_{j}\right), \quad j=1, \ldots, D, \\
& F(a)=\prod_{j=1}^{D} S_{j}(a), \quad H(a)=\prod_{j=1}^{D} I_{j}(a),
\end{aligned}
$$

where $\sum^{\prime}$ denotes the sum over monic irreducible polynomials in A. Let $\pi_{N}$ denote the number of monic irreducible polynomials in $\mathbf{A}$ of degree $N$. The prime number theorem for $\mathbf{A}$ is

$$
q^{N} / N-q^{N / 2}<\pi_{N} \leq q^{N} / N
$$

As $\operatorname{deg} \lambda_{j}=0$, by the definition of $E$ we have

$$
I_{j}(a)= \begin{cases}q^{N} / N & \text { if } \operatorname{deg} a<-d N-1, \\ \left(q^{N} / N\right) \psi\left(\operatorname{sgn}\left(a \lambda_{j}\right)\right) & \text { if } \operatorname{deg} a=-d N-1,\end{cases}
$$

and

$$
S_{j}(a)= \begin{cases}\pi_{N} & \text { if } \operatorname{deg} a<-d N-1 \\ \pi_{N} \psi\left(\operatorname{sgn}\left(a \lambda_{j}\right)\right) & \text { if } \operatorname{deg} a=-d N-1\end{cases}
$$

LEMma 2.3. If $\operatorname{deg} a \geq-d N$, then $I_{j}(a)=0$.

Proof. Since $\operatorname{deg} \lambda_{j}=0$, it suffices to show that $I(a)=0$ for $\operatorname{deg} a \geq$ $-d N$. Let $\operatorname{deg} a=-d N+l$ for some integer $l \geq 0$ and let

$$
a=a_{l} T^{-d N+l}+\ldots+a_{-1} T^{-d N-1}+a^{\prime} \in \mathbf{K}_{\infty},
$$

where $a_{j} \in \mathbb{F}_{q}, a_{l} \neq 0$, and $\operatorname{deg} a^{\prime} \leq-d N-2$. Let

$$
y=T^{N}+\sum_{j=1}^{\infty} b_{-j} T^{N-j} \in T^{N}+T^{N} \mathfrak{M}
$$

where $b_{-j} \in \mathbb{F}_{q}$. Then we have

$$
y^{d}=T^{d N}+\sum_{j=1}^{\infty}\left(d b_{-j}+c_{-j}\left(b_{-1}, \ldots, b_{-(j-1)}\right)\right) T^{d N-j},
$$


for some $c_{-j}\left(x_{1}, \ldots, x_{j-1}\right) \in \mathbb{F}_{q}\left[x_{1}, \ldots, x_{j-1}\right]$ and $c_{-1}=0$. Since $E\left(a^{\prime} y^{d}\right)$ $=1$, we have

$$
E\left(a y^{d}\right)=E\left(\left(a-a^{\prime}\right) y^{d}\right)=\psi\left(a_{-1}+\sum_{j=0}^{l} a_{j} b_{-(j+1)}^{\prime}\right)
$$

where

$$
b_{-j}^{\prime}=d b_{-j}+c_{-j}\left(b_{-1}, \ldots, b_{-(j-1)}\right) .
$$

By (7), since $2 \leq d<p$, we know that the $d$ th power mapping

$$
F: T^{N}+T^{N} \mathfrak{M} \rightarrow T^{d N}+T^{d N} \mathfrak{M}, \quad y \mapsto y^{d}
$$

is bijective and satisfies

$$
F\left(y+T^{N-(l+1)} \mathfrak{M}\right)=y^{d N}+T^{d N-(l+1)} \mathfrak{M} .
$$

By $(8),(9)$, since $\psi$ is a non-trivial additive character of $\mathbb{F}_{q}$, and $a_{l} \neq 0$, we obtain

$$
\begin{aligned}
I(a) & =\frac{1}{N} \int_{y \in T^{N}+T^{N} \mathfrak{M}} E\left(a y^{d}\right) d y=\frac{1}{N} \int_{y \in T^{N}+T^{N} \mathfrak{M}} E\left(\left(a-a^{\prime}\right) y^{d}\right) d y \\
& =\frac{1}{N} \int_{z \in T^{N-(l+1)} \mathfrak{M}} \sum_{c \in \mathbb{F}_{q}} q^{l} \cdot \psi(c) d z=0 .
\end{aligned}
$$

Let a positive integer $l$ satisfy $l \leq N / 2$ and let $y$ be a monic element in $\mathbf{K}_{\infty}$ of degree $N$. Let $\pi_{N}(y, l)$ denote the number of monic irreducible polynomials $P \in \mathbf{A}_{+}$of degree $N$ with $\operatorname{deg}(P-y)<N-l$. In [7], Corollary 2.6, or [2], Theorem 1.4, we have

$$
\pi_{N}(y, l)=\frac{q^{N-l}}{N}+O\left(q^{N / 2}\right)
$$

where the implied constant depends only on A. Given

$$
x=\sum_{i=-d N+l-1}^{-\infty} a_{i} T^{i} \in \mathbf{K}_{\infty}, \quad f=T^{d N}+\sum_{j=d N-1}^{0} f_{j} T^{j} \in \mathbf{A},
$$

where $a_{i}, f_{j} \in \mathbb{F}_{q}, a_{-d N+l-1} \neq 0$, and setting $f_{d N}=1$, we have

$$
\operatorname{Res}_{\infty}(x f)=\sum_{k=0}^{l} a_{-d N+k-1} f_{d N-k} .
$$

Let $\pi_{N, d}(f, l)$ be the number of monic irreducible polynomials $P$ of degree $N$ with $\operatorname{deg}\left(P^{d}-f\right)<d N-l$. By (7), since $2 \leq d<p$, there exists a monic element $y \in \mathbf{K}_{\infty}$ of degree $N$ satisfying $\operatorname{deg}\left(y^{d}-f\right)<d N-l$ and $\pi_{N, d}(f, l)=\pi_{N, d}\left(y^{d}, l\right)=\pi_{N}(y, l)$. Thus by (10) we get

$$
\pi_{N, d}(f, l)=\frac{q^{N-l}}{N}+O\left(q^{N / 2}\right)
$$


where the implied constant depends only on $\mathbf{A}$. If $P$ is a monic irreducible polynomial of degree $N$ satisfying $\operatorname{deg}\left(P^{d}-f\right)<d N-l$, then by (11), $\operatorname{Res}_{\infty}\left(x P^{d}\right)=\operatorname{Res}_{\infty}(x f)$. Hence combining (12), (11), and $a_{-d N+l-1} \neq 0$, we get

$\#\left\{\right.$ monic irreducibles $\left.P \mid \operatorname{deg} P=N, \operatorname{Res}_{\infty}\left(x P^{d}\right)=c\right\}$

$$
=q^{l-1}\left(\frac{q^{N-l}}{N}+O\left(q^{N / 2}\right)\right)=\frac{q^{N-1}}{N}+O\left(q^{l+N / 2}\right)
$$

for any $c \in \mathbb{F}_{q}$. Since

$$
E\left(x P^{d}\right)=\exp \left(\frac{2 \pi i \operatorname{Tr}\left(\operatorname{Res}_{\infty}\left(x P^{d}\right)\right)}{p}\right),
$$

and $\operatorname{Tr}$ is a surjective $\mathbb{F}_{p}$-linear mapping from $\mathbb{F}_{q}$ onto $\mathbb{F}_{p}$, by (13) we obtain

$$
|S(x)|=\left|\sum_{\operatorname{deg} P=N}^{\prime} E\left(x P^{d}\right)\right|=O\left(q^{l+N / 2}\right) .
$$

Therefore we have

TheOREM 2.4. Let $m$ be an integer satisfying $0 \leq m<N / 2$. Then

$$
|S(x)|=O\left(q^{m+N / 2}\right)
$$

for all $x \in \mathbf{K}_{\infty}$ with $\operatorname{deg} x=-d N+m$, where the implied constant depends only on $\mathbf{A}$.

REMARK. If $m \geq N / 2$, then the result of Theorem 2.4 is trivial.

\section{The major arcs}

Lemma 3.1. Let $n$ be a positive integer and let $-d N \leq m \leq-d N+N / 4$. Then

$$
\int_{\operatorname{deg} a \leq m}|F(a)-H(a)| \chi_{-n}(a) d a=o\left(\frac{q^{(D-d) N-N / 2-n}}{N^{D}}\right),
$$

as $N \rightarrow \infty$.

Proof. Using (5), (6), $\operatorname{sgn} \lambda_{1}+\ldots+\operatorname{sgn} \lambda_{D}=0, \chi_{-n}(a) \leq q^{-n}$, Lemma 2.3 , and (4), we obtain

$$
\begin{aligned}
& \int_{\operatorname{deg} a \leq m}|F(a)-H(a)| \chi_{-n}(a) d a \\
& \quad \leq q^{-n} \int_{\operatorname{deg} a \leq-d N-1}\left|\pi_{N}^{D}-q^{D N} / N^{D}\right| d a+q^{-n} \sum_{i=-d N}^{m} \int_{\operatorname{deg} a=i}|F(a)| d a \\
& \quad \leq O\left(\frac{q^{(D-d) N-N / 2-n}}{N^{D-1}}\right)+q^{-n} \sum_{i=-d N}^{m} \int_{\operatorname{deg} a=i}|F(a)| d a
\end{aligned}
$$


where the implied constant depends only on A. Since $S_{j}(a)=S\left(a \lambda_{j}\right)$ and $\operatorname{deg}\left(a \lambda_{j}\right)=\operatorname{deg} a$, by Theorem 2.4, we obtain

$$
\left|S_{j}(a)\right|=O\left(q^{d N+m+N / 2}\right)
$$

for $-d N \leq \operatorname{deg} a \leq m$, where the implied constant depends only on $\mathbf{A}$. As $d \geq 2$ and $D \geq 1+2^{d} \geq 5$, we obtain

$$
\begin{aligned}
q^{-n} \sum_{i=-d N}^{m} \int_{\operatorname{deg} a=i}|F(a)| d a & =O\left(q^{-n} \sum_{i=-d N}^{m} \int_{\operatorname{deg} a=i} q^{d D N+D N / 2+D m} d a\right) \\
& =O\left(q^{-n} \cdot q^{m} \cdot q^{d D N+D N / 2+D m}\right) \\
& =O\left(q^{(d D+D / 2) N+(D+1) m-n}\right) \\
& =O\left(\frac{q^{(D-d) N-N / 2-n}}{N^{D-1}}\right),
\end{aligned}
$$

where the implied constant depends only on $\mathbf{A}$.

Lemma 3.2. Let $n$ be a positive integer and let $\lambda \in \mathbf{K}_{\infty}$. Then if $m \geq$ $-d N$ and $d N>\operatorname{deg} \lambda$, we have

$$
\int_{\operatorname{deg} a \leq m} H(a) E(a \lambda) \chi_{-n}(a) d a=\frac{q^{(D-d) N-n}}{N^{D}} .
$$

Proof. By Lemma 2.3 and the definition of $H$, we have $H(a)=0$ if $\operatorname{deg} a \geq m \geq-d N$. Thus

$$
\int_{\operatorname{deg} a \geq m} H(a) E(a \lambda) \chi_{-n}(a) d a=0 .
$$

By the definitions of $H, E$ and Lemma 2.3, we have

$$
\begin{aligned}
& \int_{\mathbf{K}_{\infty}} H(a) E(a \lambda) \chi-n(a) d a \\
&=\frac{1}{N^{D}} \int_{\operatorname{deg} a<-d N} \int_{T^{N}+T^{N} \mathfrak{M}} \ldots \int_{T^{N}+T^{N} \mathfrak{M}} E\left(a\left(\lambda+\sum_{j=1}^{D} \lambda_{j} y_{j}^{d}\right)\right) \\
& \times \chi_{-n}(a) d y_{1} \ldots d y_{D} d a \\
&=\frac{1}{N^{D}} \int_{T^{N}+T^{N} \mathfrak{M}} \ldots \int_{T^{N}+T^{N} \mathfrak{M} \operatorname{deg} a<-d N} E\left(a\left(\lambda+\sum_{j=1}^{D} \lambda_{j} y_{j}^{d}\right)\right) \\
& \quad \times \chi_{-n}(a) d a d y_{1} \ldots d y_{D} .
\end{aligned}
$$


By the definition of $\chi_{-n}(a)$ and since $\operatorname{deg} a<-d N$, the above is

$$
\frac{q^{-n}}{N^{D}} \int_{T^{N}+T^{N} \mathfrak{M}} \ldots \int_{T^{N}+T^{N} \mathfrak{M} \operatorname{deg}} \int_{a<-d N} E\left(a\left(\lambda+\sum_{j=1}^{D} \lambda_{j} y_{j}^{d}\right)\right) d a d y_{1} \ldots d y_{D} .
$$

Given any $y_{1}, \ldots, y_{D} \in T^{N}+T^{N} \mathfrak{M}$, set

$$
f=\lambda+\lambda_{1} y_{1}^{d}+\ldots+\lambda_{D} y_{D}^{d}
$$

Since $d N>\operatorname{deg} \lambda, \operatorname{deg} \lambda_{j}=0$, and $\operatorname{sgn} \lambda_{1}+\ldots+\operatorname{sgn} \lambda_{D}=0$, we have $\operatorname{deg} f<d N$. This implies

$$
\int_{\operatorname{deg} a<-d N} E(a f) d a=\int_{\operatorname{deg} a<-d N} 1 d a=q^{-d N} .
$$

Therefore

$$
\int_{\mathbf{K}_{\infty}} H(a) E(a \lambda) \chi_{-n}(a) d a=\frac{q^{-n}}{N^{D}} \cdot q^{D N} \cdot q^{-d N}=\frac{q^{(D-d) N-n}}{N^{D}} .
$$

Combining these with (14), we complete the proof.

4. The minor arcs. We recall Dirichlet's theorem for $\mathbf{A}$ in

TheOREM 4.1. Given any $\alpha \in \mathbf{K}_{\infty}$ and a positive integer $N$, there exists a unique monic polynomial $Q$ and a polynomial a in $\mathbf{A}$ satisfying $(Q, a)$ $=1, \operatorname{deg} Q \leq N$, and $\operatorname{deg}(\alpha-a / Q) \leq-(\operatorname{deg} Q+N+1)$.

Proof. See [6].

For any $x \in \mathbf{K}_{\infty}$, define

$$
V(x)=\min \left\{\left|S_{1}(x)\right|,\left|S_{2}(x)\right|\right\} .
$$

Lemma 4.2. Suppose $p>d \geq 2$ and that positive numbers $\varepsilon, D_{1}$, and $\sigma_{0}$ satisfy $d-6 \varepsilon<2 D_{1}<d$. Then there exist infinitely many positive integers $N$ such that

$$
V(x) \ll q^{N} / N^{\sigma_{0}} \quad \text { for all } x \in \mathbf{K}_{\infty} \text { with }-(d-\varepsilon) N \leq \operatorname{deg} x \leq D_{1} N \text {, }
$$

where the implied constant depends only on $d, \varepsilon, D_{1}$ and $\sigma_{0}$.

Proof. Since $\lambda_{1} / \lambda_{2} \in \mathbf{K}_{\infty} \backslash \mathbf{K}$, by Theorem 4.1 there exist infinitely many monic polynomials $Q$ and polynomials $a$ in $\mathbf{A}$ such that $(Q, a)=1$ and

$$
\operatorname{deg}\left(\lambda_{1} / \lambda_{2}-a / Q\right)<-2 \operatorname{deg} Q .
$$

For a fixed pair $(Q, a)$, let $N$ be the least integer satisfying $2 \operatorname{deg} Q \leq d N$ and write

$$
\frac{\lambda_{1}}{\lambda_{2}}=\frac{a}{Q}+f \quad \text { for some } f \in \mathbf{K}_{\infty} \text { with } \operatorname{deg} f<-2 \operatorname{deg} Q .
$$


Throughout the proof of this lemma, assume that $\left(d-2 D_{1}\right) N \geq 6 d$. Given any $x \in \mathbf{K}_{\infty}$ satisfying $-(d-\varepsilon) N \leq \operatorname{deg} x \leq D_{1} N$, let $m$ denote the least integer satisfying $\left(5 d+2 D_{1}\right) N / 6 \leq m$. For any $j=1,2$, again by Theorem 4.1 there exist monic polynomials $Q_{1}, Q_{2}$ and polynomials $a_{1}, a_{2}$ such that

$$
\operatorname{deg}\left(x \lambda_{j}-a_{j} / Q_{j}\right)<-\operatorname{deg} Q_{j}-m, \quad j=1,2,
$$

where $\left(Q_{j}, a_{j}\right)=1$ and $\operatorname{deg} Q_{j} \leq m$. Since $\operatorname{deg} \lambda_{j}=0, \operatorname{deg}\left(x \lambda_{j}\right)=\operatorname{deg} x \geq$ $-(d-\varepsilon) N$. Combining this with $(17)$ and $m>(d-\varepsilon) N$ because $d-6 \varepsilon<2 D_{1}$, we have $a_{j} \neq 0$ and we can write

$$
x \lambda_{j}=\frac{a_{j}}{Q_{j}}+\frac{f_{j}}{Q_{j}}=\frac{a_{j}}{Q_{j}}\left(1+\frac{f_{j}}{a_{j}}\right) \quad \text { for some } f_{j} \in \mathbf{K}_{\infty} \text { with } \operatorname{deg} f_{j}<-m .
$$

Thus

$$
\frac{\lambda_{1}}{\lambda_{2}}=\frac{x \lambda_{1}}{x \lambda_{2}}=\frac{Q_{2} a_{1}}{Q_{1} a_{2}}\left(1+\frac{f_{1}}{a_{1}}\right)\left(1+\frac{f_{2}}{a_{2}}\right)^{-1} .
$$

Since $\operatorname{deg} \lambda_{1}=\operatorname{deg} \lambda_{2}=0$, we have $\operatorname{deg}\left(Q_{2} a_{1}\right)=\operatorname{deg}\left(Q_{1} a_{2}\right)$. We may write

$$
\frac{\lambda_{1}}{\lambda_{2}}=\frac{Q_{2} a_{1}}{Q_{1} a_{2}}+f_{3} \quad \text { for some } f_{3} \in \mathbf{K}_{\infty} \text { with } \operatorname{deg} f_{3}<-m \text {. }
$$

By (16), and since $0<m \leq d(N-1)$ because $\left(d-2 D_{1}\right) N \geq 6 d$, we have

$$
\operatorname{deg}\left(\frac{a}{Q}-\frac{Q_{2} a_{1}}{Q_{1} a_{2}}\right)<-m
$$

This implies

$$
\operatorname{deg}\left(a_{2} Q_{1} a-Q_{2} a_{1} Q\right)<d N / 2-m+\operatorname{deg}\left(Q_{1} a_{2}\right) .
$$

If $a_{2} Q_{1} a-Q_{2} a_{1} Q \neq 0$, then $\operatorname{deg}\left(Q_{1} a_{2}\right)>-d N / 2+m$. If $a_{2} Q_{1} a-Q_{2} a_{1} Q=0$, then

$$
\frac{a}{Q}=\frac{Q_{2} a_{1}}{Q_{1} a_{2}}
$$

Since $(Q, a)=1$ and $d \geq 2, \operatorname{deg}\left(Q_{1} a_{2}\right) \geq \operatorname{deg} Q>d(N-1) / 2 \geq-d N / 2+m$ because $\left(d-2 D_{1}\right) N \geq 6 d$. Thus we always have $\operatorname{deg}\left(Q_{1} a_{2}\right)>-d N / 2+m$. Since $\operatorname{deg}\left(x \lambda_{2}-a_{2} / Q_{2}\right)<-\operatorname{deg} Q_{2}-m,-(d-\varepsilon) N \leq \operatorname{deg} x \leq D_{1} N, \operatorname{deg} \lambda_{2}$ $=0$, and $m>(d-\varepsilon) N$, we have $D_{1} N \geq \operatorname{deg} x=\operatorname{deg} x \lambda_{2}=\operatorname{deg}\left(a_{2} / Q_{2}\right)$. Combining these, we have

$$
\operatorname{deg}\left(Q_{1} Q_{2}\right)=\operatorname{deg}\left(Q_{1} a_{2}\right)+\operatorname{deg}\left(Q_{2} / a_{2}\right)>-d N / 2+m-D_{1} N .
$$

This implies that $\max \left\{\operatorname{deg} Q_{1}, \operatorname{deg} Q_{2}\right\}>-d N / 4+\left(m-D_{1} N\right) / 2$. Without loss of generality, assume that $\operatorname{deg} Q_{1}>-d N / 4+\left(m-D_{1} N\right) / 2$. By the definition of $m$, we have

$$
\operatorname{deg} Q_{1}+m>-\frac{d N}{4}+\frac{m-D_{1} N}{2}+m \geq d N .
$$


Combining this with (17), we obtain

$$
S_{1}(x)=S\left(x \lambda_{1}\right)=S\left(a_{1} / Q_{1}\right) .
$$

Set $\sigma=(d N-m) / \ln N$. Since $m-1<\left(5 d+2 D_{1}\right) N / 6$ and $d>2 D_{1}$, we have

$$
\sigma>\frac{\left(d-2 D_{1}\right) N-6}{6 \ln N} \geq d 2^{6 d}\left(\sigma_{0}+1\right)
$$

for large $N$. Since

$$
\sigma \ln N=d N-m<\operatorname{deg} Q_{1} \leq m=d N-\sigma \ln N
$$

by Theorem 4.3 below, we obtain

$$
\left|S_{1}(x)\right|=\left|S\left(x \lambda_{1}\right)\right|=\left|S\left(a_{1} / Q_{1}\right)\right| \ll q^{N} / N^{\sigma_{0}}
$$

for large $N$. Thus there exist infinitely many positive integers $N$ such that

$$
V(x) \ll q^{N} / N^{\sigma_{0}} \quad \text { for all }-(d-\varepsilon) N \leq \operatorname{deg} x \leq D_{1} N .
$$

Now we recall three theorems proved in [9]. They are used in the proof of Lemma 4.6 and in the proofs of polynomial Waring and polynomial WaringGoldbach problems (cf. [4] and [9]).

Theorem 4.3. Let $2 \leq d<p$ and let $\sigma_{0} \geq 0$. Suppose that $(Q, a)=1$, $\sigma \ln N \leq \operatorname{deg} Q \leq d N-\sigma \ln N$. Then, if $\sigma \geq d 2^{6 d}\left(\sigma_{0}+1\right)$, we have

$$
|S(a / Q)| \ll q^{N} / N^{\sigma_{0}}
$$

where the implied constant depends only on $d, \sigma_{0}$, and $q$.

Proof. See [9], Theorem 11.8.

Theorem 4.4 (Hua's lemma). Suppose that $1 \leq d<p$. Then

$$
\int_{\mathfrak{M}}\left|\sum_{x \in \mathbf{A}_{+}, \operatorname{deg} x=N} E\left(x^{d} a\right)\right|^{2^{d}} d a \ll N^{C} q^{N\left(2^{d}-d\right)}
$$

for some $C$, where the implied constant and the constant $C$ depend on $d$ and A, but not on $N$. In other words, the number of solutions of

$$
x_{1}^{d}+\ldots+x_{2^{d-1}}^{d}=y_{1}^{d}+\ldots+y_{2^{d-1}}^{d}
$$

with $x_{i}, y_{i} \in \mathbf{A}_{+}$and $\operatorname{deg} x_{i}=\operatorname{deg} y_{i}=N$ is $\ll N^{C} q^{N\left(2^{d}-d\right)}$.

Proof. See [9], Theorem 4.2.

Remark. In [4], Theorem 8.13, the right-hand side of (18) is $q^{N\left(2^{d}-d+\varepsilon\right)}$. Following Hua's idea (cf. [10], Theorem 4), we improve this to the form of Theorem 4.4 . 
Theorem 4.5. Suppose $d \geq 9$ and $s \geq 2 d^{2} \ln d+d^{2} \ln \ln d+2 d^{2}-2 d$. Then

$$
\int_{\mathfrak{M}}\left|\sum_{x \in \mathbf{A}_{+}, \operatorname{deg} x=N} E\left(x^{d} a\right)\right|^{2 s} d a \ll q^{N(2 s-d)},
$$

where the implied constant depends only on $d, s$, and $q$. In other words, the number of solutions of

$$
x_{1}^{d}+\ldots+x_{s}^{d}=y_{1}^{d}+\ldots+y_{s}^{d}
$$

with $x_{i}, y_{i} \in \mathbf{A}_{+}$and $\operatorname{deg} x_{i}=\operatorname{deg} y_{i}=N$ is $\ll q^{N(2 s-d)}$.

Proof. See [9], Theorem 7.5.

Lemma 4.6. Let $D, n$ be positive integers and let $d, \varepsilon, D_{1}$ and $N$ be as in Lemma 4.2. Then, if $D_{1} N \geq n$ and

$$
D \geq \begin{cases}1+2^{d} & \text { if } 2 \leq d<11 \\ 2\left[2 d^{2} \ln d+d^{2} \ln \ln d+2 d^{2}-2 d\right]+1 & \text { if } d \geq 11\end{cases}
$$

we have

$$
\int_{-(d-\varepsilon) N \leq \operatorname{deg} a}|F(a)| \chi_{-n}(a) d a \ll q^{(D-d) N} / N^{\sigma_{0}}
$$

for any positive number $\sigma_{0}$, where the implied constant depends only on $D$, $d, \varepsilon, D_{1}, \sigma_{0}$, and the constant $C$ of Theorem 4.4.

Proof. By the definition of $\chi_{-n}$, we know that $\chi_{-n}(a)=0$ if $\operatorname{deg} a \geq n$. Thus $\chi_{-n}(a)=0$ if $\operatorname{deg} a \geq D_{1} N$. Thus

$$
\int_{-(d-\varepsilon) N \leq \operatorname{deg} a}|F(a)| \chi_{-n}(a) d a=\int_{-(d-\varepsilon) N \leq \operatorname{deg} a \leq D_{1} N}|F(a)| \chi_{-n}(a) d a .
$$

If $V(a)=\min \left\{\left|S_{1}(a)\right|,\left|S_{2}(a)\right|\right\}$, then

$$
|F(a)| \leq V(a)\left(\left|S_{1}(a) \prod_{j=3}^{D} S_{j}(a)\right|+\left|S_{2}(a) \prod_{j=3}^{D} S_{j}(a)\right|\right) .
$$

This implies

$$
|F(a)| \leq V(a)\left(\sum_{j=1}^{D}\left|S_{j}(a)\right|^{D-1}\right)
$$

Since

$$
D \geq \begin{cases}1+2^{d} & \text { if } 2 \leq d<11 \\ 2\left[2 d^{2} \ln d+d^{2} \ln \ln d+2 d^{2}-2 d\right]+1 & \text { if } d \geq 11\end{cases}
$$

and $\operatorname{deg} \lambda_{j}=0,\left|S_{j}(a)\right| \leq q^{N}$, we have 


$$
\begin{aligned}
\int_{\mathbf{K}_{\infty}}\left|S_{j}(a)\right|^{D-1} \chi_{-n}(a) d a & \\
& \leq \begin{cases}q^{N\left(D-1-2^{d}\right)} \int_{\mathbf{K}_{\infty}}|S(a)|^{2^{d}} \chi_{-n}(a) d a & \text { if } 2 \leq d<11, \\
q^{N(D-2 s-1)} \int_{\mathbf{K}_{\infty}}|S(a)|^{2 s} \chi_{-n}(a) d a & \text { if } d \geq 11,\end{cases}
\end{aligned}
$$

where $s=\left[2 d^{2} \ln d+d^{2} \ln \ln d+2 d^{2}-2 d\right]$. By Lemma 2.2, the last integral is equal to the number of monic irreducible $2 s$-tuples $\left(P_{1}, \ldots, P_{2 s}\right)$ such that $\operatorname{deg} P_{i}=N$ and

$$
\operatorname{deg}\left(\sum_{i=1}^{s}\left(P_{i}^{d}-P_{s+i}^{d}\right)\right)<-n .
$$

Since $n>0$, this integral is equal to the number of monic irreducible $2 s$ tuples $\left(P_{1}, \ldots, P_{2 s}\right)$ such that $\operatorname{deg} P_{i}=N$ and

$$
\sum_{i=1}^{s}\left(P_{i}^{d}-P_{s+i}^{d}\right)=0 .
$$

Using (19) and Theorems 4.4 and 4.5, we obtain

$$
\int_{\mathbf{K}_{\infty}}\left|S_{j}(a)\right|^{D-1} \chi_{-n}(a) d a \ll \begin{cases}N^{C} q^{N(D-d-1)} & \text { if } 2 \leq d<11, \\ q^{N(D-d-1)} & \text { if } d \geq 11 .\end{cases}
$$

Combining these with Lemma 4.2 (substitute $\sigma_{0}+C$ for $\sigma_{0}$ ), we obtain

$$
\begin{aligned}
\int_{-(d-\varepsilon) N \leq \operatorname{deg} a}|F(a)| \chi_{-n}(a) d a & \\
& \leq \int_{-(d-\varepsilon) N \leq \operatorname{deg} a \leq D_{1} N} V(a) \sum_{j=1}^{D}\left|S_{j}(a)\right|^{D-1} \chi_{-n}(a) d a \\
& \ll \frac{q^{N}}{N^{\sigma_{0}+C}} \cdot N^{C} q^{N(D-d-1)}=\frac{q^{(D-d) N}}{N^{\sigma_{0}}} .
\end{aligned}
$$

5. Completion of the proof of the main theorem. We conclude the proof of Theorem 2.1 by collecting the above results. First of all, Lemma 3.2 with $\varepsilon>0$ and a positive integer $n$ gives

$$
\int_{\operatorname{deg} a \leq-(d-\varepsilon) N} H(a) E(a \lambda) \chi_{-n}(a) d a=q^{(D-d) N-n} / N^{D},
$$

as $d N>\operatorname{deg} \lambda$. Combining this with Lemma 3.1, when $0<\varepsilon<1 / 4$ and $n=[m \ln N]$, we have

$$
\int_{\operatorname{deg} a \leq-(d-\varepsilon) N} F(a) E(a \lambda) \chi_{-[m \ln N]}(a) d a \gg q^{(D-d) N} / N^{D+m},
$$


as $N \rightarrow \infty$. In Lemmas 4.2 and 4.6 , if $\varepsilon, D_{1}, d, D, n$ and $\sigma_{0}$ satisfy $d-6 \varepsilon<$ $2 D_{1}<d, 2 \leq d<p, \sigma_{0}=D+m+1, n=[m \ln N], D_{1} N \geq n$, and

$$
D \geq \begin{cases}1+2^{d} & \text { if } 2 \leq d<11 \\ 2\left[2 d^{2} \ln d+d^{2} \ln \ln d+2 d^{2}-2 d\right]+1 & \text { if } d \geq 11\end{cases}
$$

then there are infinitely many positive integers $N$ (note that these $N$ come from $\left.\lambda_{1} / \lambda_{2} \in \mathbf{K}_{\infty} / \mathbf{K}\right)$ such that

$$
\int_{-(d-\varepsilon) N \leq \operatorname{deg} a} F(a) E(a \lambda) \chi_{-[m \ln N]}(a) d a \ll q^{(D-d) N} / N^{D+m+1} .
$$

Therefore, taking $\varepsilon=1 / 6, D_{1}=d / 2-1 / 4$ and combining (20) and (21), we see that for any positive integer $m$,

$$
\begin{aligned}
\int_{\mathbf{K}_{\infty}} \sum_{\operatorname{deg} P_{1}=N}^{\prime} \cdots & \sum_{\operatorname{deg} P_{D}=N}^{\prime} E\left(a\left(\lambda+\sum_{i=1}^{D} \lambda_{i} P_{i}^{d}\right)\right) \chi_{-[m \ln N]}(a) d a \\
& =\int_{\mathbf{K}_{\infty}} F(a) E(a \lambda) \chi_{-[m \ln N]}(a) d a \gg q^{(D-d) N} / N^{D+m} .
\end{aligned}
$$

It follows from Lemma 2.2 that there exist infinitely many positive integers $N$ for which there are $\gg q^{(D-d) N} / N^{D+m} D$-tuples $\left(P_{1}, \ldots, P_{D}\right)$ of monic irreducible polynomials with $\operatorname{deg} P_{i}=N$ and

$$
\operatorname{deg}\left(\lambda+\lambda_{1} P_{1}^{d}+\ldots+\lambda_{D} P_{D}^{d}\right)<-m \ln N+1 .
$$

This completes the proof of Theorem 2.1 .

\section{References}

[1] A. Baker, On some diophantine inequalities involving primes, J. Reine Angew. Math. 228 (1967), 166-181.

[2] M. Car, Distribution des polynômes irréductibles dans $\mathbb{F}_{q}[T]$, Acta Arith. 88 (1999), 141-153.

[3] H. Davenport and H. Heilbronn, On indefinite quadratic forms in five variables, J. London Math. Soc. 21 (1946), 185-193.

[4] G. W. Effinger and D. R. Hayes, Additive Number Theory of Polynomials Over a Finite Field, Clarendon Press, Oxford, 1991.

[5] G. Harman, Diophantine approximation by prime numbers, J. London Math. Soc. (2) 44 (1991), 218-226.

[6] D. R. Hayes, The expression of a polynomial as a sum of three irreducibles, Acta Arith. 11 (1966), 461-488.

[7] C. N. Hsu, The distribution of irreducible polynomials in $\mathbb{F}_{q}[t]$, J. Number Theory 61 (1996), 85-96.

[8] —, Diophantine inequalities for polynomial rings, ibid. 78 (1999), 46-61.

[9] - On polynomial Waring-Goldbach problem, preprint.

[10] L. K. Hua, Additive Theory of Prime Numbers, Amer. Math. Soc., Providence, RI, 1965. 
[11] K. Ramachandra, On the sums $\sum_{j=1}^{K} \lambda_{j} f_{j}\left(p_{j}\right)$, J. Reine Angew. Math. 262-263 (1973), 158-165.

[12] W. Schwarz, Über die Lösbarkeit gewisser Ungleichungen durch Primzahlen, ibid. 212 (1963), 150-157.

[13] R. C. Vaughan, Diophantine approximation by prime numbers II, Proc. London Math. Soc. (3) 28 (1974), 385-401.

Department of Mathematics

National Taiwan Normal University

88 Sec. 4 Ting-Chou Road

Taipei, Taiwan

E-mail: maco@math.ntnu.edu.tw

Received on 25.10.1999

and in revised form on 19.6.2000 